\title{
A Mathematical Analysis of Fractional Fragmentation Dynamics with Growth
}

\author{
Emile Franc Doungmo Goufo \\ Department of Mathematical Sciences, University of South Africa, Florida Science Campus, Gauteng 0003, South Africa
}

Correspondence should be addressed to Emile Franc Doungmo Goufo; dgoufef@unisa.ac.za

Received 21 April 2014; Accepted 25 July 2014; Published 7 August 2014

Academic Editor: Gestur Ólafsson

Copyright (C) 2014 Emile Franc Doungmo Goufo. This is an open access article distributed under the Creative Commons Attribution License, which permits unrestricted use, distribution, and reproduction in any medium, provided the original work is properly cited.

\begin{abstract}
We make use of the theory of strongly continuous solution operators for fractional models together with the subordination principle for fractional evolution equations (Bazhlekova (2000) and Prüss (1993)) to analyze and show existence results for a fractional fragmentation model with growth characterized by its growth rate $r$. Indeed, strange phenomena like the phenomenon of shattering (McGrady and Ziff (1987)) and the sudden appearance of infinite number of particles in some systems with initial finite particles number could not be fully explained by classical models of fragmentation or aggregation. Then, there is an increasing volition to try new approaches and extend classical models to fractional ones. In the growth model, one of the major challenges in the analysis occurs when $1 / r(x)$ is integrable at $x_{0} \geq 0$, the minimum size of a cell. We restrict our analysis to the case of integrability of $r^{-1}$ at $x_{0}$. This case needs more considerations on the boundary condition, which, in this paper, is the McKendrick-von Foerster renewal condition. In the process, some properties of Mittag-Leffler relaxation function Berberan-Santos (2005) are exploited to finally prove that there is a positive solution operator to the full model.
\end{abstract}

\section{Model's Motivation and Introduction}

Despite its three centuries of age, fractional calculus remains lightly unpopular amongst science and engineering community. However, there is a growing interest in extending analysis involving normal calculus with integer orders to noninteger orders (real or complex order) [1-4] because its applications have attracted a great range of attention in the past few years. Most of classical models used are formulated as initial or boundary value problems for dynamical evolution equations taking the form

$$
\frac{d}{d t} u(t)=Q u(t),
$$

where $t$ is the time taken from $\mathbb{R}_{+}$and $Q$ is an operator in a Banach space. The problem is to find the state $u(t)$ of the system at a time $t>t_{0}$ depending on the initial state $u\left(t_{0}\right)$ at the initial time $t_{0}$. The normal derivative $d / d t$ is seen as the representation of the rate of accumulation or loss in the system, that is, gain rate minus loss rate, at infinitesimal bounded space. However, there are three essential motivations for considering here the fractional fragmentation model with growth.

(1) Classical models of clusters' fission with normal derivative $d / d t$ cannot fully explain strange phenomena like the sudden appearance of an infinite number of particles in some systems which contained at the beginning a finite particles number and the phenomenon of shattering [5]. The latter is seen as an explosive or dishonest Markov process (see, for example, $[6,7]$ ) and has been associated with an infinite cascade of breakup events creating a "dust" of particles of zero size which, however, carry nonzero mass.

(2) As said above, $d / d t$ is seen as the representation of the rate of change (accumulation or loss) in the system, considered at infinitesimal bounded space. But sometime the infinitesimal space contains traps (of various sizes) where the variable under study is temporarily parked then; will the $d / d t$ replicate the real picture of accumulation or loss? Similarly these trap pictures 
could be islands or forbidden zones in the infinitesimal space where the variable (particle, mass, density, flux, etc.) cannot reside; accordingly, the rate of accumulation or loss will be different than $d / d t$. Hence, the fractional differentiation $d^{\gamma} / d t^{\gamma}$, with $\gamma \in \mathbb{R}$ or $\mathbb{C}$, may give the sub- or superrate of accumulation or loss with index $\gamma$ representing the heterogeneity distribution of the infinitesimal space (traps or islands)! However, substituting $d / d t$ by the fractional derivative $D_{t}^{\gamma}$ requires some considerations as we will in the following sections.

(3) Most of works on fragmentation with normal derivative $d / d t$ have been performed under assumption that the ensemble of particles is well mixed so that the particle distribution is uniform in space. However, recent approach using individual based models [8] yields in a natural way to systems in which splitting particles are distributed in space according to some prescribed probability density, leading, however, to models with explicit space dependence. We note that similar models were also considered earlier in [9] but with emphasis only on well-posedness.

These reasons are the sources of the increasing volition to try new approaches and extend classical models to models with fractional derivative and investigate them with various and different techniques in order to establish broader outlooks on the real phenomena they describe. For instance, we have differential equations with fractional orders increasingly used to model many problems in applied sciences including engineering, applied mathematics, physics, biology, chemistry, economic, and other domains of applications. In the process, differential equations with fractional derivative have become a useful tool for describing nonlinear phenomena of science and engineering models. In the same way, the process of fragmentation of clusters occurs in many branches of natural sciences ranging from physics to chemistry, engineering, biology, ecology, and numerous domains of applied sciences, such as the depolymerization, the rock fractures, and of break of droplets.

With this in mind, we obtain the following fractional model of fragmentation process with growth:

$$
\begin{aligned}
D_{t}^{\gamma}( & u(x, t)) \\
= & -a(x) u(x, t)+\int_{x+x_{0}}^{\infty} a(y) b(x \mid y) u(y, t) d y \\
& -\partial_{x}[r(x) u(x, t)],
\end{aligned}
$$

where $x \geq x_{0}, 0<\gamma<1, t>0$, and $D_{t}^{\gamma}$ is given as

$$
D_{\tau}^{\gamma}(u(x, \tau))=-\lim _{t \rightarrow 0} \frac{\mathfrak{g}_{\gamma}(t) u(x, \tau)-u(x, \tau)}{t}
$$

with $\mathfrak{g}_{\gamma}(t)$ the fractional time evolution, considered as universal attractor semigroups of coarse grained macroscopic time evolutions $[10,11]$. It is shown that $[10,12]$

$$
\begin{array}{r}
D_{\tau}^{\gamma}(u(x, \tau))=-\frac{1}{\Gamma(-\gamma)} \int_{0}^{\infty} \frac{u(x, \tau-r)-u(x, \tau)}{r^{\gamma+1}} d r, \\
0<\gamma<1,
\end{array}
$$

which is the fractional derivative of $u(x, t)$ in the sense of Michaud; see [12]. The full description of this model is done in the next section.

Recall that fission models of type (2) with "normal" time derivative of order one $\partial / \partial t$ have been comprehensively analyzed in numerous works (see, e.g., [13-21]). Conservative and nonconservative regimes for fragmentation equations have been thoroughly investigated; see $[5,15]$, and, in particular, the breach of the mass conservation law (called shattering) has been attributed to a phase transition creating a dust of "zero-size" particles with nonzero mass, which are beyond the model's resolution. Shattering can be interpreted from the probabilistic point of view as the explosion in the Markov process describing fragmentation [21] and from an analytic point of view as dishonesty of the semigroup associated with the model. Transport-type models with convection were investigated in [17] where the author showed that the convection part does not affect the breach of the conservation laws. In [15], the authors studied the nonlocal fragmentation and showed that the process is conservative if at infinity daughter particles tend to go back into the system with a high known probability.

As said earlier, in the model with growth, one of the major problems in the analysis occurs when $1 / r(x)$ is integrable at $x_{0} \geq 0$ which is the minimum size of a cell. In this paper, the analysis is restricted to the case where $r^{-1}$ is integrable at $x_{0}$. This case needs more considerations on the boundary condition, namely, the McKendrick-von Foerster renewal condition. In the investigation, some properties of Mittag-Leffler relaxation function are exploited to show that there is a positive solution operator to the full model.

\section{Model Description and Settings}

2.1. Classical Fragmentation Differential Equation with Growth. To describe the dynamics here, we use the cluster (or group) density function $u(x, t)$. Here $x \in\left[x_{0}, \infty\right)$ is a variable that represents the size of the cluster, $x_{0} \geq 0$ is the minimum single cell size, the variable $t$ represents time, and $u(x, t)$ is the concentration of groups of size $x$ at time $t$. We assume that for each $t \geq 0$ the function $x \mapsto u(x, t)$ is from the space

$$
\begin{aligned}
Y_{1} & =L_{1}\left(\left[x_{0}, \infty\right), x d x\right) \\
& =\left\{\psi:\|\psi\|_{1}:=\int_{x_{0}}^{\infty} x|\psi(x)| d x<\infty\right\} .
\end{aligned}
$$

This space $Y_{1}$ is the most appropriate one because $\int_{x_{0}}^{\infty} x|\psi(x)| d x$ is the total number of cells in the population. 
Clusters grow as a result of divisions of phytoplankton cells. The growth rate is denoted by $r$. We assume that $r$ is a nonnegative function, differentiable at $x_{0}$ and

$$
r \in A C\left(\left(x_{0}, \infty\right)\right) \cap X_{\infty},
$$

where $X_{\infty}$ is the dual space of $Y_{1}$ and $r \in A C\left(\left(x_{0}, \infty\right)\right)$ means that $r$ is absolutely continuous in the standard sense on each compact subinterval of $\left(x_{0}, \infty\right)$. We denote by $\|\cdot\|_{\infty}$ the norm of $X_{\infty}$ and we recall that

$$
\|\psi\|_{\infty}=\operatorname{ess} \sup _{x_{0} \leq x<\infty} \frac{|\psi(x)|}{x},
$$

and the duality pairing is the normal integral

$$
\langle\psi, \omega\rangle=\int_{x_{0}}^{\infty} \psi(x) \omega(x) d x
$$

During a small time interval $\Delta t$, a fraction $a(x) \Delta t$ of the clusters of size $x$ are undergoing breakup, that is, $a$ is the fragmentation rate. We assume that it is a nonnegative function and

$$
a \in L_{\infty, \text { loc }}\left(\left(x_{0}, \infty\right)\right) .
$$

The size distribution of daughter particles after fragmentation is denoted by $b$. We assume that

$$
\int_{x_{0}}^{y-x_{0}} x b(x \mid y) d x=y, \quad y>2 x_{0}
$$

which accounts for mass conservation after any fragmentation event. Thus, classical model of the dynamics of fragmentation with growth is given by the integrodifferential equation:

$$
\begin{aligned}
\frac{\partial}{\partial t} u(x, t)= & -a(x) u(x, t) \\
& +\int_{x+x_{0}}^{\infty} a(y) b(x \mid y) u(y, t) d y \\
& -\partial_{x}[r(x) u(x, t)] .
\end{aligned}
$$

The boundary condition considered here is the McKendrick-von Foerster renewal condition:

$$
\lim _{x \rightarrow x_{0}^{+}} r(x) u(x, t)=\int_{x_{0}}^{\infty} \beta(y) u(y, t) d y,
$$

where $\beta \in X_{\infty}$. The function $\beta(y)$ describes the number of single cells that fall off a cluster of size $y$ and join the single cell population [22]. The boundary condition represents the addition of newborn single cells to the single cell population.

2.2. Abstract Reformulation. The analysis is performed in the space $Y_{1}$. We denote by $\mathscr{T}$ and $\mathscr{B}$ the expressions appearing on the right-hand side of (11); that is,

$$
\begin{aligned}
& {[\mathscr{T} \psi](x)=-\frac{d}{d x}[r(x) \psi(x)]-a(x) \psi(x)} \\
& {[\mathscr{B} \psi](x)=\int_{x+x_{0}}^{\infty} a(y) b(x \mid y) \psi(y) d y .}
\end{aligned}
$$

$\mathscr{T}$ and $\mathscr{B}$ are defined on measurable and finite almost everywhere functions $\psi$ for which they make pointwise (almost everywhere) sense. For each fixed $t \geq 0$, we define a function $u(t):\left(x_{0}, \infty\right) \rightarrow \mathbb{R}$ of the "mass" variable $x$ by

$$
u(t)(x)=u(x, t), \quad \text { for a.e. } x>x_{0}, t \geq 0 .
$$

Hence, $u$ is the function from $[0, \infty)$ into the space $Y_{1}$. Since $Y_{1}$ is a Banach space of type $L, D_{t}^{\gamma} u$ can be thought of as the fractional derivative with respect to $t$ of the function $u$ : $[0, \infty) \rightarrow Y_{1}$ defined by (15). For fixed $t>0$, we can write the right-hand side of $(11)$ as $(\mathscr{T}+\mathscr{B}) u(t)$ defined on its maximal domain. The initial condition can therefore be written as $u(0)=u_{0}$ instead of $u(x, 0)=u_{0}(x)$.

A vital role in the analysis of the model is played by the integrability of $1 / r(x)$ at $x_{0}$. Indeed, if $1 / r(x)$ is integrable at $x_{0}$, the characteristics do reach the line $x=x_{0}$ and therefore the boundary condition becomes crucial for the uniqueness investigation. If not, the characteristics do not reach the line $x=x_{0}$ and the prescription of a boundary condition is of no use. Therefore, we consider only the case of integrability of $1 / r(x)$.

\section{Solution Operator for the Fractional Growth Problem with $r^{-1}$ Integrable at $x_{0}$}

We are interested in investigating the fractional differential model given by

$$
\begin{aligned}
D_{t}^{\gamma} u(x, t) & =[T+B] u(x, t), \quad x \geq x_{0}, 0<\gamma<1, t>0 \\
u(0) & =u_{0},
\end{aligned}
$$

where $T$ is the realization of $\mathscr{T}$ (defined via (13)) on $Y_{1}, B$ as the realization of $\mathscr{B}$ (see (14)) on the domain

$$
\begin{aligned}
& D(T) \\
& \quad=\left\{\psi \in Y_{1} ; q \psi \in Y_{1}, r \psi \in A C\left(\left(x_{0}, \infty\right)\right),(r \psi)_{x} \in Y_{1}\right\}
\end{aligned}
$$

and $D_{t}^{\gamma}$ is given as

$$
\begin{array}{r}
D_{\tau}^{\gamma}(u(x, \tau))=-\lim _{t \rightarrow 0} \frac{\mathfrak{g}_{\gamma}(t) u(x, \tau)-u(x, \tau)}{t}, \\
x \geq x_{0}, \quad 0<\gamma<1, \quad t>0
\end{array}
$$

with $\mathfrak{g}_{\gamma}(t)$, the fractional time evolution, considered as universal attractor semigroups of coarse grained macroscopic time evolutions $[10,11]$. It is shown that $[10,12]$

$$
\begin{array}{r}
D_{\tau}^{\gamma}(u(x, \tau))=-\frac{1}{\Gamma(-\gamma)} \int_{0}^{\infty} \frac{u(x, \tau-r)-u(x, \tau)}{r^{\gamma+1}} d r, \\
0<\gamma<1,
\end{array}
$$

which is the fractional derivative of $u(x, t)$ in the sense of Michaud; see [12]. However, substituting $\partial_{t}$ by $D_{t}^{\gamma}$ in (11) is justified in the sense that the presence of $\partial_{t}$ in (11) reflects 
a basic symmetry of the time translation invariance and the basic principle of locality. Indeed, from the relation

$$
\frac{d}{d \tau} g(\tau)=\lim _{t \rightarrow 0} \frac{g(\tau)-g(\tau-t)}{t}=-\lim _{t \rightarrow 0} \frac{\mathfrak{g}(t) g(\tau)-g(\tau)}{t},
$$

we see that $-d / d t$ is identified as the infinitesimal generator of time translation $\mathfrak{g}(t) g(\tau)=g(\tau-t)$. Hence, this considers $\mathfrak{g}(t)$ as the expression of the general time evolution, which is the same consideration done in the definitions (18) and (19) of $D_{t}^{\gamma}$. Thus, the derivative of fractional order $D_{t}^{\gamma}, 0<\gamma<1$ was found to be, in general, infinitesimal generator of coarse grained macroscopic time evolution. It is shown [23] that all macroscopic time evolutions have fractional derivatives, with order less than unity, as their infinitesimal generators. Therefore, in Proposition 4 below, we provide a relation between the generator $D_{t}^{\gamma}, 0<\gamma<1$ of the macroscopic time evolution and $d / d t$.

Since $1 / r(x)$ is integrable at $x_{0}$, the characteristics do reach the line $x=x_{0}$ and we have to restrict the operator $T$ to a domain in which the boundary condition (12) is satisfied. In this respect, we introduce $T_{\beta}$ as the restriction of $T$ to

$$
\begin{aligned}
D & \left(T_{\beta}\right) \\
& =\left\{\psi \in D(T): \lim _{x \rightarrow x_{0}^{+}} r(x) \psi(x)=\int_{x_{0}}^{\infty} \beta(y) \psi(y) d y\right\} .
\end{aligned}
$$

Considering now $B$ as the restriction (via (14)) of $\mathscr{B}$ to $D\left(T_{\beta}\right)$, the model (16) becomes

$$
\begin{aligned}
D_{t}^{\gamma} u(x, t) & =\left[T_{\beta}+B\right] u(x, t), \quad x \geq x_{0}, 0<\gamma<1, t>0 \\
u(0) & =u_{0}
\end{aligned}
$$

with $D(T)$ and $D_{t}^{\gamma}$ conserving the same definition as in (16).

Lemma 1. $\left(T_{\beta}+B, D\left(T_{\beta}\right)\right)$ is a well-defined operator.

Proof. It is obvious to see that $D\left(T_{\beta}\right) \subseteq D(B)$. Indeed, let $\psi \in$ $D\left(T_{\beta}\right)$; then by definition of $D\left(T_{\beta}\right)$, it is clear that $a \psi \in Y_{1}$. It follows that

$$
\begin{aligned}
\|B \psi\|_{1} & =\int_{x_{0}}^{\infty}\left|\int_{x}^{\infty} a(y) b(x \mid y) \psi(y) d y\right| x d x \\
& \leq \int_{x_{0}}^{\infty}|\psi(y)| a(y)\left(\int_{x_{0}}^{y} b(x \mid y) x d x\right) d y \\
& \leq \int_{x_{0}}^{\infty}|\psi(y)| a(y) y d y \\
& =\|a \psi\|_{1}<\infty
\end{aligned}
$$

where we used (10). Therefore, $\psi \in D(B)$.

To proceed we need the following definition.
Definition 2. Consider an operator $Q$ applying in the fractional model

$$
D_{t}^{\gamma}(u(x, t))=Q u(x, t), \quad 0<\gamma<1, t>0, x \geq x_{0}, t>0
$$

subject to the initial condition

$$
u(x, 0)=f(x), \quad x \geq x_{0}
$$

and defined in the Banach space $X_{1}$. A family $\left(G_{Q}(t)\right)_{t>0}$ of bounded operators on $X_{1}$ is called a solution operator of the fractional Cauchy problem (24)-(25) if

(i) $G_{Q}(0)=I_{X_{1}}$;

(ii) $G_{Q}(t)$ is strongly continuous for every $t \geq 0$;

(iii) $Q G_{Q}(t) f=G_{Q}(t) Q f$ for all $f \in D(Q)$;

(iv) $G_{Q}(t) D(Q) \subset D(Q)$;

(v) $G_{Q}(t) f$ is a (classical) solution of the model (24)-(25) for all $f \in D(Q), t \geq 0$.

It is well known $[14,17]$ that an operator $\widetilde{Q} \in \mathscr{G}(M, \omega)$ means $\widetilde{Q}$ generates a $C_{0}$-semigroup $\left(G_{\widetilde{Q}}(t)\right)_{t>0}$ so that there exist $M>0$ and $\omega$ such that

$$
\left\|G_{\widetilde{Q}}(t)\right\| \leq M e^{\omega t} .
$$

Whence, by analogy if the fractional Cauchy problem (24)(25) has a solution operator $\left(G_{Q}(t)\right)_{t>0}$ verifying (26), then we say that $Q \in \mathscr{G}^{\gamma}(M, \omega)$. The solution operator $\left(G_{Q}(t)\right)_{t>0}$ is contractive if

$$
\left\|G_{Q}(t)\right\|_{X_{1}} \leq 1
$$

and we say $Q \in \mathscr{G}^{\gamma}(1,0)$.

Note that if we have a contraction solution operator, we can use Definition 2 to identify the fractional Cauchy problem of which it is a solution. Usually, however, we are interested in the reverse question, that is, in finding the solution operator, that is, contractive, for a given fractional model. The answer is given by the following theorem (seen as an analogue of Hille-Yosida theorem).

Theorem 3. An operator $A_{\gamma} \in \mathscr{G}^{\gamma}(1,0)$ for $0<\gamma \leq 1$ if and only if

(a)

$$
(0, \infty) \subset \rho\left(A_{\gamma}\right)
$$

(b)

$$
\lambda^{\gamma-1} R\left(\lambda^{\gamma}, A_{\gamma}\right) f=\int_{0}^{\infty} e^{-\lambda r} G_{\gamma}(r) f d r
$$

with $\lambda>0, f \in X_{1}$, where $\left(G_{y}(t)\right)_{t>0}$ is a family of strongly continuous operators satisfying $(27)$ and $\rho\left(A_{\gamma}\right)$ is the resolvent set of the operator $A_{\gamma}$

$$
\begin{gathered}
\rho\left(A_{\gamma}\right)=\left\{\lambda \in \mathbb{R} ; \lambda I-A_{\gamma}: D\left(A_{\gamma}\right) \longrightarrow Y_{1}\right. \text { is invertible } \\
\text { and } \left.\left(\lambda I-A_{\gamma}\right)^{-1} \text { bounded and linear }\right\} .
\end{gathered}
$$


Proof. This theorem is a particular version of [24, Theorem 1.3] and the proof follows the same steps.

In our analysis we will need some interesting properties of the Mittag-Leffler relaxation function $E_{\gamma}(-x)$ which arises in the description of complex relaxation processes and that corresponds to a relaxation function when $x$ is a positive real number like the time variable. We have that (see $[25,26])$

$$
E_{\gamma}[z]=\sum_{n=0}^{\infty} \frac{z^{n}}{\Gamma(n \gamma+1)}
$$

where $\Gamma$ is the Gamma function. Using the Laplace transform $\mathscr{L}$ and its inverse, it can be proved (see [27-29]) that

$$
\begin{aligned}
E_{\gamma}(-x) & =\frac{2 x}{\pi} \int_{0}^{\infty} \frac{E_{2 \gamma}\left(-r^{2}\right)}{x^{2}+r^{2}} d r, \quad 0<\gamma<1, \\
& =\frac{\sin (\gamma \pi)}{\pi} \int_{0}^{\infty} \frac{r^{\gamma-1} e^{-x^{1 / \gamma} r} d r}{r^{2 \gamma}+2 r^{\gamma} \cos (\gamma \pi)+1}, \quad 0<\gamma<1 \\
& =\int_{0}^{\infty} \sum_{n=0}^{\infty} \frac{(-r)^{n} e^{-x r} d r}{n ! \Gamma(1-\gamma-\gamma n)}, \quad 0<\gamma<1 \\
& =\frac{1}{2 \pi i} \int_{\Upsilon} e^{\left(r-x r^{\gamma}\right)} r^{\gamma-1} e^{-x r} d r, \quad 0<\gamma<1,
\end{aligned}
$$

where $Y$ is a contour domain which encircles the origin counterclockwise, going from $-\infty$ to $-\infty$.

Now, we consider the full operator in (22) as a perturbation of the following Cauchy problem representing the loss part of the fractional fission process:

$$
\begin{aligned}
D_{t}^{\gamma}(u(x, t)) & =-a(x) u(x, t)(=A u(x, t)), \quad 0<\gamma<1, \\
u(x, 0) & =f(x), \quad x \geq x_{0}, t>0 .
\end{aligned}
$$

Take

$$
f \in D(A)
$$

we can use the point of view of $C_{0}$-semigroup theory to say that the operator $A$ is the infinitesimal generator of a $C_{0}$ semigroup $\left(G_{A}(t)\right)_{t \geq 0}$, from the original semigroup $(G(t))_{t \geq 0}$ (the multiplication semigroup) generated by the differentiation operator $-d / d t$ and obtained via a simple integration as

$$
(G(t) u)(x, t)=e^{-a(x) t} u(x, t) .
$$

The existence of $(G(t))_{t \geq 0}$ comes from assumption that the fission rate satisfies (9). Therefore, the infinitesimal generators of $\left(G_{A}(t)\right)_{t \geq 0}$ may be interpreted [30] as the distributions, evaluated on the right time translation group, which leads to the following proposition.
Proposition 4. Consider $A=D_{t}^{\gamma}, 0<\gamma<1$; let $u \in D(A)$. The infinitesimal generators $A$ of $C_{0}$-semigroups $\left(G_{A}(t)\right)_{t \geq 0}$ are related to $d / d t$ by the representations

$$
\begin{aligned}
& -\left(-\frac{d}{d t}\right)^{\gamma} u(x, t) \\
& =-D_{t}^{\gamma}(u(x, t)) \\
& =\lim _{\varepsilon \rightarrow 0+} \frac{1}{\Gamma(-\gamma)} \int_{\varepsilon}^{\infty} r^{-\gamma-1}\left[1-e^{-a(x) r}\right] u(x, t) d r .
\end{aligned}
$$

Proof. We exploit the Marchaud type representation (19) of infinitesimal generators (see also $[12,31]$ ). Let $\delta$ be the Dirac measure; then if we take $C_{0}^{1}(\mathbb{R})$ as the set of test functions on $\mathbb{R}$, we know that (see [32]) the fractional derivative distribution $\delta^{\gamma}, 0<\gamma<1$, of $\delta$ can be expressed by

$$
\left\langle\varphi, \delta^{\gamma}\right\rangle=\frac{1}{-\Gamma(-\gamma)} \int_{0}^{\infty} r^{-\gamma-1}[\varphi(x)-\varphi(x+r)] d r
$$

and approximated by the family $\left(\mathscr{F}_{\varepsilon}^{\gamma}\right)_{\varepsilon>0}$ of finite Borel measure on $\mathbb{R}_{+}$given as

$$
\begin{array}{r}
\left\langle\varphi, \mathscr{F}_{\varepsilon}^{\gamma}\right\rangle=\frac{1}{-\Gamma(-\gamma)} \int_{\varepsilon}^{\infty} r^{-\gamma-1}[\varphi(x)-\varphi(x+r)] d r, \\
\forall \varphi \in C_{0}^{1}(\mathbb{R}) .
\end{array}
$$

The family $\left(\mathscr{F}_{\varepsilon}^{\gamma}\right)_{\varepsilon>0}$ generates the bounded linear operators $\beth_{\mathscr{F} \gamma}^{\gamma}$ defined by

$$
\begin{array}{r}
I_{\mathscr{F}_{\varepsilon} \gamma} u(x, t)=\frac{1}{-\Gamma(-\gamma)} \int_{\mathcal{\varepsilon}}^{\infty} r^{-\gamma-1}[u(x, t)-G(r) u(x, t)] d r, \\
u \in Y_{1},
\end{array}
$$

with $G$ defined in (35). Using Laplace transform $\mathscr{L}$, we can show (see [33]) that

$$
\begin{aligned}
\mathscr{L} & \left(\beth_{\mathscr{F}_{\varepsilon}^{\gamma}}\right)(x) \\
& =x^{\gamma} \cdot \mathscr{L}\left[\frac{1}{-\Gamma(-\gamma) \Gamma(1+\gamma)}\left(\left(\frac{\dot{-}}{\varepsilon}\right)_{+}^{\gamma}-\left(\frac{\dot{-}}{\varepsilon}+1\right)_{+}^{\gamma}\right)\right](x),
\end{aligned}
$$$$
x \geq x_{0} \text {, }
$$

which yields, by uniqueness theorems for Laplace transformation and well known properties of convolution operator $*$,

$$
\mathscr{F}_{\varepsilon}^{\gamma}=\delta^{\gamma} *\left[\frac{1}{-\Gamma(-\gamma) \Gamma(1+\gamma)}\left(\left(\frac{\dot{-}}{\varepsilon}\right)_{+}^{\gamma}-\left(\frac{\dot{-}}{\varepsilon}+1\right)_{+}^{\gamma}\right)\right]
$$

and then

$$
\begin{gathered}
A \int_{0}^{\infty} \frac{1}{-\Gamma(-\gamma) \Gamma(1+\gamma)}\left(\left(\frac{r}{\varepsilon}\right)_{+}^{\gamma}-\left(\frac{r}{\varepsilon}+1\right)_{+}^{\gamma}\right) G(r) u(x, t) d r \\
\quad=\frac{1}{-\Gamma(-\gamma)} \int_{\varepsilon}^{\infty} r^{-\gamma-1}[u(x, t)-G(r) u(x, t)] d r .
\end{gathered}
$$


Now taking $\varepsilon \rightarrow 0$, the assertion follows by using the fact that $A$ is a closed operator [34] and

$$
\begin{gathered}
\lim _{\varepsilon \rightarrow 0} \int_{0}^{\infty} \frac{1}{-\Gamma(-\gamma) \Gamma(1+\gamma)}\left(\left(\frac{r}{\varepsilon}\right)_{+}^{\gamma}-\left(\frac{r}{\varepsilon}+1\right)_{+}^{\gamma}\right) \\
\times G(r) u(x, t) d r=u(x, t) .
\end{gathered}
$$

Theorem 5. There is an extension of the operator $T_{\beta}+B$, say $K_{\beta} \in \mathscr{G}^{\gamma}\left(1,\left(\|r\|_{\infty}+x_{0}\|\beta\|_{\infty}\right)^{1 / \gamma}\right)$, such that there is a solution operator $\left(G_{K_{\beta}}(t)\right)_{t \geq 0}$ for the fractional model (22) that is positive and then satisfies

$$
\left\|G_{K_{\beta}}(t)\right\| \leq e^{\left(\|r\|_{\infty}+x_{0}\|\beta\|_{\infty}\right)^{1 / \gamma} t}
$$

Proof. We make use of the subordination principle developed in $[24,35]$. Consider the classical model

$$
\begin{aligned}
\frac{d}{d t} u(x, t) & =\left[T_{\beta}+B\right] u(x, t) \\
u(0) & =u_{0},
\end{aligned}
$$

where $T_{\beta}$ and $B$ are defined via (21) and (14), respectively. Then, by [36, Theorem 2], there is an extension $\widetilde{K_{\beta}}$ of the operator $T_{\beta}+B$ which generates a positive semigroup $\left(G_{\widetilde{K}_{\beta}}(t)\right)_{t \geq 0}$ satisfying

$$
\left\|G_{\widetilde{K_{\beta}}}(t)\right\| \leq e^{\left(\|r\|_{\infty}+x_{0}\|\beta\|_{\infty}\right) t} .
$$

Now, using the Definition 2 , we say $\widetilde{K_{\beta}} \in \mathscr{G}\left(1,\|r\|_{\infty}+\right.$ $\left.x_{0}\|\beta\|_{\infty}\right)$. Exploiting the relaxation relation (32), we set

$$
G_{K_{\beta}}(t) f=\int_{0}^{\infty} \Theta_{\gamma}(t, r) G_{\widetilde{K_{\beta}}}(r) f d r,
$$

where

$$
\Theta_{\gamma}(t, r)=\frac{1}{t^{\gamma}} \sum_{n=0}^{\infty} \frac{(-r)^{n} t^{-\gamma n}}{n ! \Gamma(1-\gamma-\gamma n)} .
$$

We aim to show that $G_{K_{\beta}}(t)$ is the solution operator for the fractional model (22) and is positive. The second last relation of (32) and monotonicity of $E_{\gamma}(-x)$ imply that $\left(G_{K_{\beta}}(t)\right)_{t>0}$ is positive. Finally, by the subordination principle developed in $[24,35]$, we have $\widetilde{K_{\beta}} \in \mathscr{G}^{\gamma}\left(1,\left(\|r\|_{\infty}+x_{0}\|\beta\|_{\infty}\right)^{1 / \gamma}\right)$ since $\widetilde{K_{\beta}} \in$ $\mathscr{G}\left(1,\|r\|_{\infty}+x_{0}\|\beta\|_{\infty}\right)$. This shows that $G_{K_{\beta}}(t)$ given by (47) verifies $\left\|G_{K_{\beta}}(t)\right\| \leq e^{\left(\|r\|_{\infty}+x_{0}\|\beta\|_{\infty}\right)^{1 / \gamma} t}$. Thus by Theorem 3 , $G_{K_{\beta}}(t)$ is the solution operator for the fractional model (22), that is, positive, and satisfies (44).

\section{Concluding Remarks}

Under the McKendrick-von Foerster renewal condition (12), we have set conditions allowing us to analyze and show the existence of a positive solution operator to the fission model
(22) with growth and derivative of fractional order $\gamma$, with $0<\gamma<1$. We have made use of the theory of strongly continuous solution operators, properties of Mittag-Leffler relaxation function, and the subordination principle. Growth fragmentation equations with fractional order dynamics has never been studied before and this work improves the preceding ones with the inclusion of the transport (growth) and the derivative of order less than unity. Whence, the theory analyzed in this paper is viewed as an opening step that will lead to the full characterization of the operator $K_{\gamma}$ under which the fractional model admits solution operators and, maybe, leads to a better understanding of strange phenomena like the phenomenon of shattering and the sudden appearance of an infinite number of particles in some systems with initial finite particles number.

\section{Conflict of Interests}

The author declares that there is no conflict of interests regarding the publication of this paper.

\section{References}

[1] M. Caputo, "Linear models of dissipation whose Q is almost frequency independent: part II," Journal of Royal Astronomical Society, vol. 13, pp. 529-539, 1967.

[2] K. B. Oldham and J. Spanier, The Fractional Calculus, Academic Press, New York, NY, USA, 1999.

[3] I. Podlubny, Fractional Differential Equations, Academic Press, San Diego, Calif, USA, 1999.

[4] K. S. Miller and B. Ross, An Introduction to the Fractional Calculus and Fractional Differential Equations, John Wiley \& Sons, New York, NY, USA, 1993.

[5] E. D. McGrady and R. M. Ziff, “"Shattering” transition in fragmentation," Physical Review Letters, vol. 58, no. 9, pp. 892-895, 1987.

[6] W. J. Anderson, Continuous-Time Markov Chains. An Applications-Oriented Approach, Springer Series in Statistics: Probability and its Applications, Springer, New York, NY, USA, 1991.

[7] J. R. Norris, Markov Chains, Cambridge University Press, Cambridge, UK, 1998.

[8] R. Rudnicki and R. Wieczorek, "Phytoplankton dynamics: from the behaviour of cells to a transport equation," Mathematical Modelling of Natural Phenomena, vol. 1, no. 1, pp. 83-100, 2006.

[9] M. Lachowicz and D. Wrzosek, "A nonlocal coagulation-fragmentation model," Applicationes Mathematicae, vol. 27, no. 1, pp. 45-66, 2000.

[10] A. V. Balakrishnan, "Fractional powers of closed operators and the semigroups generated by them," Pacific Journal of Mathematics, vol. 10, pp. 419-437, 1960.

[11] K. Yosida, Fonctional Analysis, Springer, Berlin, Germany, 6th edition, 1980.

[12] S. G. Samko, A. A. Kilbas, and O. I. Marichev, Franctional Integrals and Derivatives. Theory and Application, Gordon and Breach, Amsterdam, The Netherlands, 1993.

[13] E. F. Doungmo Goufo and S. C. Oukouomi Noutchie, "Global analysis of a discrete nonlocal and nonautonomous fragmentation dynamics occurring in a moving process," Abstract and Applied Analysis, vol. 2013, Article ID 484391, 9 pages, 2013. 
[14] J. Banasiak and L. Arlotti, Perturbations of Positive Semigroups with Applications, Springer Monographs in Mathematics, Springer, 2006.

[15] E. F. Doungmo Goufo and S. C. Oukouomi Noutchie, "Honesty in discrete, nonlocal and randomly position structured fragmentation model with unbounded rates," Comptes Rendus Mathematique, vol. 351, no. 19-20, pp. 753-759, 2013.

[16] S. C. Oukouomi Noutchie and E. F. Doungmo Goufo, "On the honesty in nonlocal and discrete fragmentation dynamics in size and random position," ISRN Mathematical Analysis, vol. 2013, Article ID 908753, 7 pages, 2013.

[17] S. C. Oukouomi Noutchie and E. F. Doungmo Goufo, "Global solvability of a continuous model for nonlocal fragmentation dynamics in a moving medium," Mathematical Problems in Engineering, vol. 2013, Article ID 320750, 8 pages, 2013.

[18] C. R. Garibotti and G. Spiga, "Boltzmann equation for inelastic scattering," Journal of Physics. A. Mathematical and General, vol. 27, no. 8, pp. 2709-2717, 1994.

[19] A. Majorana and C. Milazzo, "Space homogeneous solutions of the linear semiconductor Boltzmann equation," Journal of Mathematical Analysis and Applications, vol. 259, no. 2, pp. 609629, 2001.

[20] R. M. Ziff and E. D. McGrady, "The kinetics of cluster fragmentation and depolymerisation," Journal of Physics A, vol. 18, no. 15, pp. 3027-3037, 1985.

[21] W. Wagner, "Explosion phenomena in stochastic coagulationfragmentation models," The Annals of Applied Probability, vol. 15, no. 3, pp. 2081-2112, 2005.

[22] A. S. Ackleh, "Parameter estimation in a structured algal coagulation-fragmentation model," Nonlinear Analysis: Theory, Methods \& Applications, vol. 28, no. 5, pp. 837-854, 1997.

[23] R. Hilfer, "On new class of phase transitions," in Random Magnetism High-Temperature Superconductivity, p. 85, World Scientific, Singapore, 1994.

[24] J. Prüss, Evolutionary Integral Equations and Applications, vol. 87 of Monographs in Mathematics, Birkhäuser, Basel, Switzerland, 1993.

[25] G. M. Mittag-Leffler, "Sur la nouvelle fontion $E_{\alpha}(x)$," Comptes Rendus Hebdomadaires des Séances de l'Académie des Sciences, vol. 137, pp. 554-558, 1903.

[26] A. Érdelyi, W. Magnus, F. Oberhettinger, and F. G. Tricomi, Higher Transcendental Functions, vol. 3, McGraw-Hill, New York, NY, USA, 1955.

[27] M. N. Berberan-Santos, "Properties of the Mittag-Leffler relaxation function," Journal of Mathematical Chemistry, vol. 38, no. 4, pp. 629-635, 2005.

[28] R. Gorenflo, Y. Luchko, and F. Mainardi, "Analytical properties and applications of the Wright function," Fractional Calculus and Applied Analysis, vol. 2, no. 4, pp. 383-414, 1999.

[29] E. M. Wright, “The generalized Bessel function of order greater than one," The Quarterly Journal of Mathematics. Oxford. Second Series, vol. 11, pp. 36-48, 1940.

[30] I. Gel'fand and G. Shilov, Generalized Functions, vol. 1, Academic Press, New York, NY, USA, 1964.

[31] J. L. Lions and J. Peetre, "Sur une classe d'espace d'interpolation," Publications Mathématiques de l'Institut des Hautes Études Scientifiques, vol. 19, pp. 5-68, 1964.

[32] B. Rubin, Fractional Integrals and Potentials, Addison Wesley Longman, Harlow, UK, 1996.
[33] U. Westphal, "ein Kalkül für gebrochene Potenzen infinitesimaler Erzeuger von Halbgruppen und Gruppen von Operatoren, Teil I: Halbgruppen-erzeuger," Compositio Mathematica, vol. 22, pp. 67-103, 1970.

[34] K. Engel and R. Nagel, One-Parameter Semigroups for Linear Evolution Equations, vol. 194 of Graduate Texts in Mathematics, Springer, New York, NY, USA, 2000.

[35] E. G. Bazhlekova, "Subordination principle for fractional evolution equations," Fractional Calculus \& Applied Analysis, vol. 3, no. 3, pp. 213-230, 2000.

[36] J. Banasiak, S. C. Oukouomi Noutchie, and R. Rudnicki, "Global solvability of a fragmentation-coagulation equation with growth and restricted coagulation," Journal of Nonlinear Mathematical Physics, vol. 16, supplement 1, pp. 13-26, 2009. 


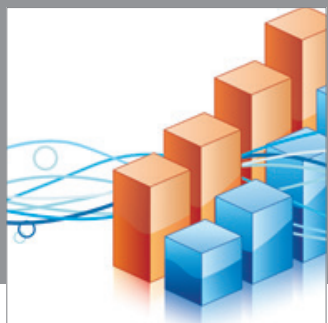

Advances in

Operations Research

mansans

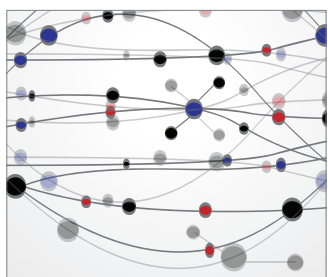

The Scientific World Journal
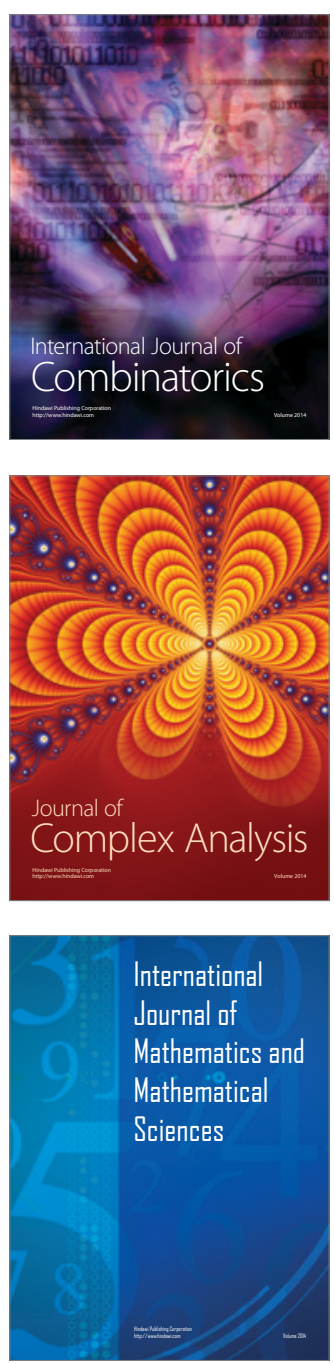
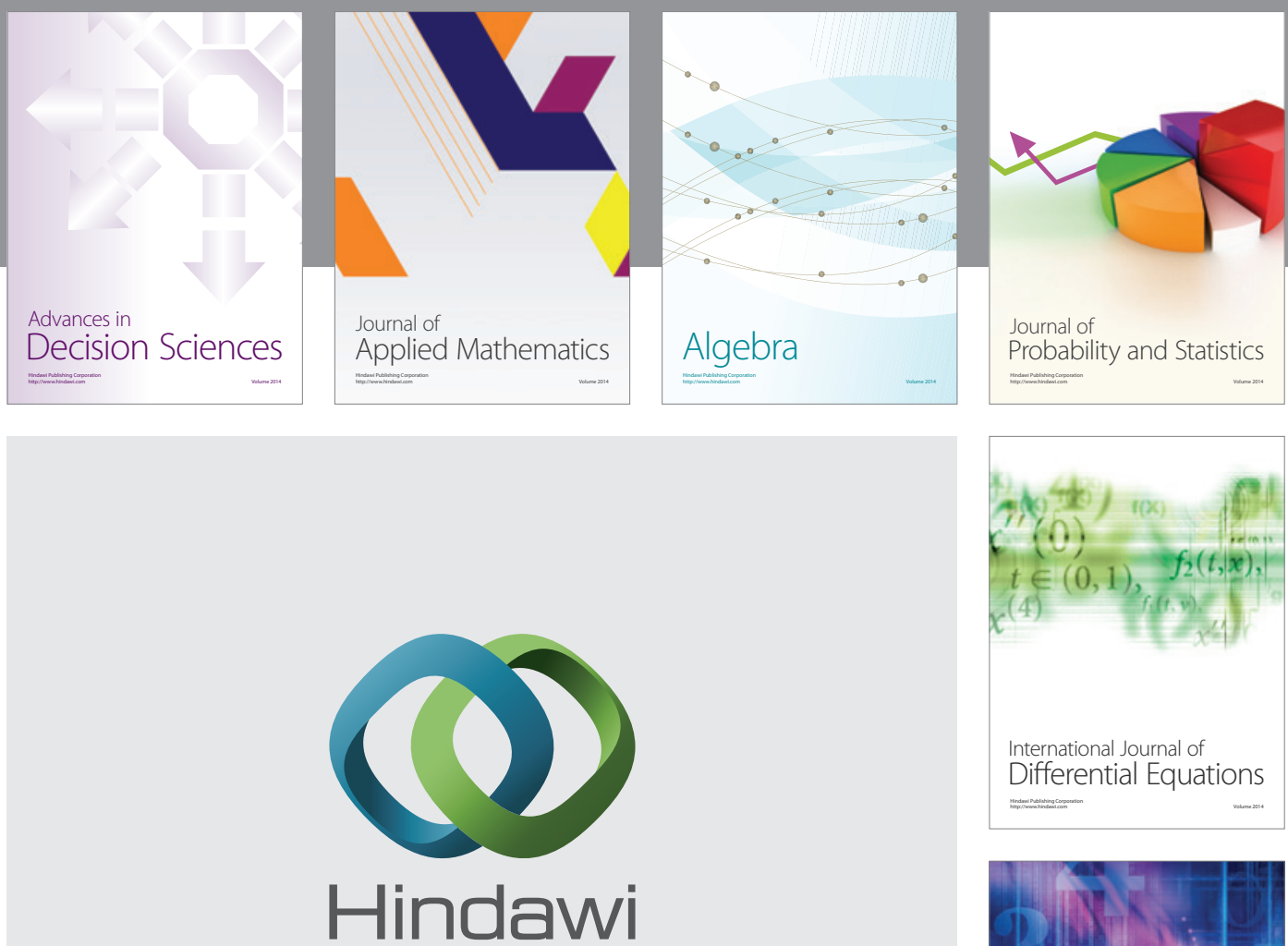

Submit your manuscripts at http://www.hindawi.com
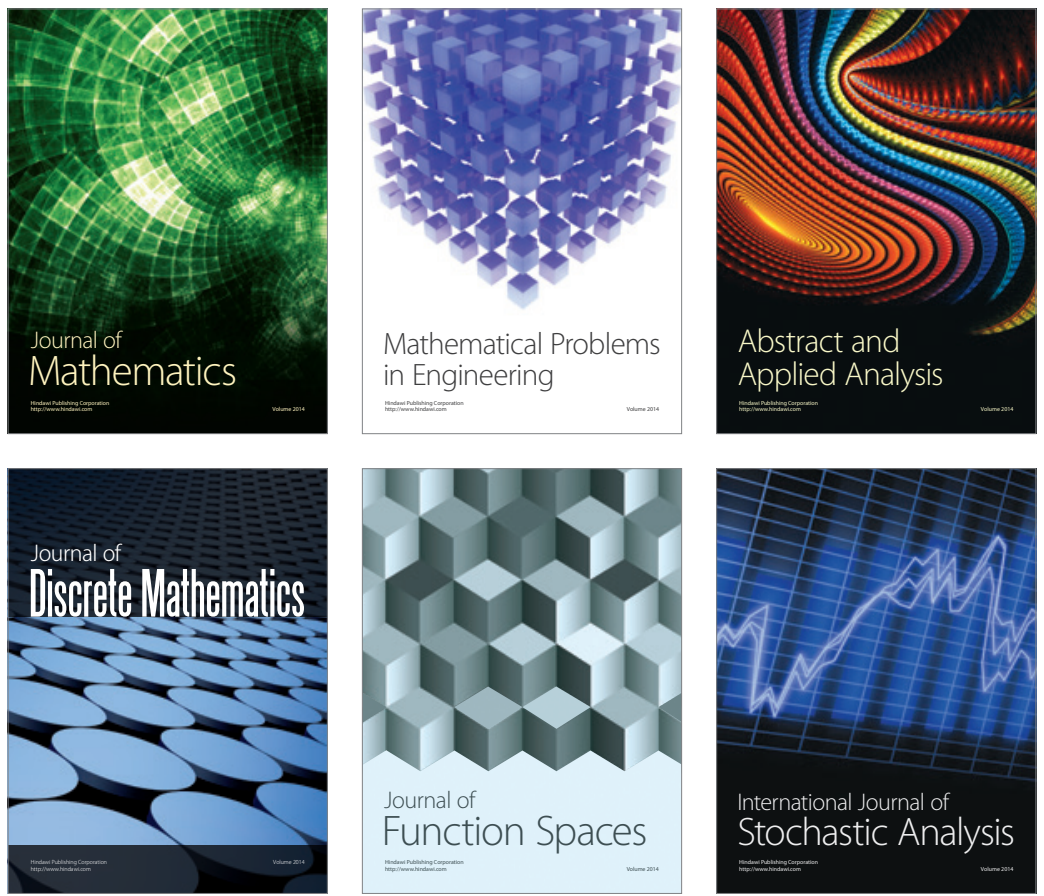

Journal of

Function Spaces

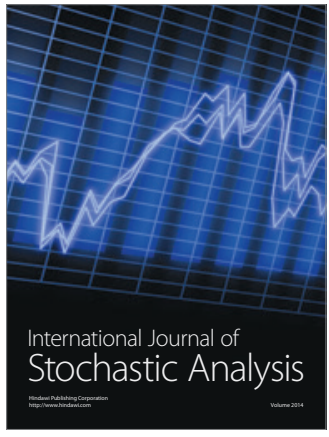

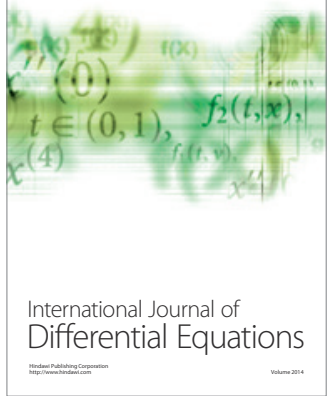
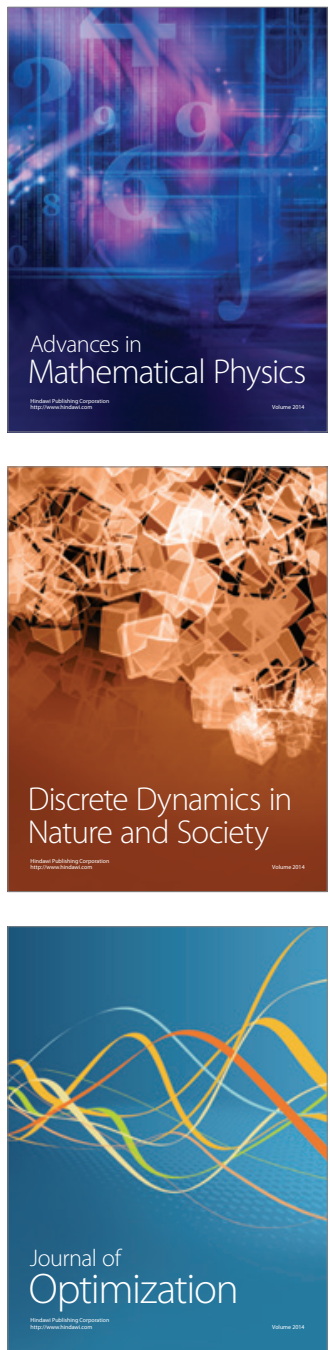